\section{A confluência entre o "adequado" e o "saudável": análise da instituição da noção de alimentação adequada e saudável nas políticas públicas do Brasil}

\author{
A convergence of "adequate" and "healthy": \\ an analysis of the notion of adequate and \\ healthy diet in public policies in Brazil
}

\author{
La confluencia entre lo "adecuado" y lo \\ "saludable": análisis de la noción de lo que \\ se considera alimentación adecuada y saludable \\ en las políticas públicas de Brasil
}

Janaína Braga de Paiva 1,2 Lílian Miranda Magalhães 2 Sandra Maria Chaves dos Santos 2 Ligia Amparo da Silva Santos 2 Leny Alves Bomfim Trad 1

\title{
Resumo
}

No âmbito das políticas públicas brasileiras para alimentação e nutrição e para segurança alimentar e nutricional, a partir da virada do século XXI, observa-se que a alimentação deixou de ser adjetivada somente como "saudável" e passou também a ser referida como "adequada". Essas noções vêm sendo construidas e defendidas socio-historicamente e passando por ampliações e ressignificações ao longo do tempo. Propôs-se neste estudo analisar como se instituiu a expressão "alimentação adequada e saudável" no Brasil. Foi realizada uma análise documental tendo em vista elucidar o desenvolvimento conceitual dos termos em questão no contexto brasileiro. Foram analisados documentos institucionais, a exemplo dos textos originais dos campos da segurança alimentar e nutricional e da alimentação e nutrição. Observa-se uma transição no modo de pensar a alimentação saudável no campo da alimentação e nutrição, antes centrada no estatuto do nutriente. Atualmente, tratamos de uma compreensão ampliada sobre a noção de alimentação, que a partir do debate sobre a superação do foco no componente nutricional dos alimentos, inclui a compreensão sobre um direito humano fundamental, bem como a compreensão sobre as questões socioculturais e afetivas do comer e sobre as questões da sustentabilidade ambiental da produção de alimentos com base em modelos produtivos pautados em princípios agroecológicos e da soberania alimentar. Argumenta-se que a combinação entre as expressões é representativa do esforço em aproximar as compreensões e os debates dos campos da segurança alimentar e nutricional e da alimentação e nutrição no que se refere à polissêmica noção de alimentação.

Dieta Saudável; Segurança Alimentar e Nutricional; Direitos Humanos

\author{
Correspondência \\ J. B. Paiva \\ Instituto de Saúde Coletiva, Universidade Federal da Bahia. \\ Rua Basílio da Gama 316, Campus Canela, Salvador, BA \\ 40110-907, Brasil. \\ janainapaivanutri@gmail.com \\ 1 Instituto de Saúde Coletiva, Universidade Federal da Bahia, \\ Salvador, Brasil. \\ 2 Escola de Nutrição, Universidade Federal da Bahia, Salvador, \\ Brasil.
}




\section{Introdução}

No âmbito das políticas públicas brasileiras para alimentação e nutrição e para segurança alimentar e nutricional, a partir da virada do século XXI, observa-se que a alimentação deixou de ser adjetivada somente como "saudável” e passou também a ser referida como "adequada”. Assim, nas diversas publicações científicas e institucionais a expressão "alimentação saudável” foi gradativamente substituída por "alimentação adequada e saudável”. É partindo dessa constatação que questionamos: "Como se deu a conjunção entre os dois termos que conformam a contemporânea noção de 'alimentação adequada e saudável'?”. "E quais as suas implicações para o campo da Alimentação e Nutrição no Brasil?”.

A “alimentação adequada e saudável” é uma terminologia genuinamente brasileira. Não há descritor indexado que una os termos healthy eating e adequate food, sendo assim, tal forma de mencionar e conceber a alimentação não está presente nas publicações internacionais produzidas em outros países. Considerando a relevância da língua inglesa para a produção científica mundial, destacamos ainda que a alimentação saudável é referida pelas seguintes palavras-chave: healthy eating, healthy food, healthy diet, balanced diets. E, para indicar o direito humano à alimentação adequada: right to food $\mathrm{e}$ human right to adequate food.

Diante disso, propõe-se neste estudo analisar como se instituiu a expressão "alimentação adequada e saudável” no Brasil. Contextualizaremos a trajetória de cada uma das expressões que a compõem, vinculando-a aos desdobramentos do campo científico-político de âmbito internacional, bem como aos aspectos políticos e sociais do cenário brasileiro que subsidiaram a associação entre as mesmas. Argumentaremos que a expressão "alimentação adequada e saudável” emerge de iniciativas de ampliação e ressignificação de noções acerca da alimentação, construídas e defendidas socio-historicamente.

Para tanto, foi realizada uma análise documental tendo em vista elucidar o desenvolvimento conceitual dos termos em questão no contexto brasileiro. O Brasil tem um longo histórico de políticas públicas voltadas à superação de carências nutricionais e, mais recentemente, à superação da epidemia de sobrepeso/obesidade. Contudo, a primeira publicação institucional relevante sobre alimentação e nutrição só aconteceu na década de 1950. Considerando esse marco temporal, foram consultados e lidos na íntegra documentos oficiais produzidos a partir da segunda metade do século XX. A análise dos documentos foi guiada pelos seguintes objetivos: precisar quando as expressões "alimentação adequada" e "alimentação saudável" foram incorporadas nas publicações institucionais brasileiras; identificar as concepções ou definições que embasam as respectivas referências ao termo; discutir as implicações decorrentes da difusão de ambos os termos.

Os textos originais foram acessados em sites do Ministério da Saúde (http://www.saude.gov.br/) e do Conselho Nacional de Segurança Alimentar e Nutricional (CONSEA. http://www4.planalto. gov.br/consea/). Aqueles documentos que não estavam disponíveis on-line foram solicitados junto ao setor responsável dentro do Ministério da Saúde. Assim, foram analisados em ordem cronológica os seguintes documentos: A Conjuntura Alimentar e o Problema da Nutrição no Brasil: Plano Geral de Trabalho 1953/54 1; Decreto no 72.0342; Decreto no 77.1163; Relatório Final da I Conferência Nacional de Alimentação e Nutrição - 8a Conferência Nacional de Saúde - CNS 4; Relatório Final da I Conferência Nacional de Segurança Alimentar - CNSA 5; Publicação do Conselho Nacional de Segurança Alimentar e Nutricional (CONSEA) sobre a I Conferência Nacional de Segurança Alimentar 6; 1a Política Nacional de Alimentação e Nutrição (1 $\mathrm{a}$ e $2 \underline{a}$ edições) 7,8; Relatório Final da II Conferência Nacional de Segurança Alimentar e Nutricional - CNSAN 9; Lei no 11.346 (Lei Orgânica de Segurança Alimentar e Nutricional - LOSAN, 2006) 10; Guia Alimentar para a População Brasileira: Promovendo a Alimentação Saudável 11; Relatório Final da III Conferência Nacional de Segurança Alimentar e Nutricional 12; Glossário Temático: Alimentação e Nutrição 13; Decreto no 7.27214; Política Nacional de Promoção da Saúde 15; Emenda Constitucional no 64 16; Relatório Final da IV Conferência Nacional de Segurança Alimentar e Nutricional 17; 2a Política Nacional de Alimentação e Nutrição 18; Glossário Temático: Alimentação e Nutrição (2a edição) 19; Guia Alimentar para a População Brasileira (2a edição) 20; Relatório Final da V Conferência Nacional de Segurança Alimentar e Nutricional 21; Plano Nacional de Segurança Alimentar e Nutricional - PLANSAN (2012-2015) 22 e Plano Nacional de Segurança Alimentar e Nutricional - PLANSAN (2016-2019) 23.

O presente texto está dividido em duas principais sessões. A primeira discute o contexto internacional no que se refere às noções de alimentação adequada e de alimentação saudável, considerando a sua relação com os campos da segurança alimentar e nutricional e da alimentação e nutrição, respec- 
tivamente. Em seguida, com base em uma perspectiva histórica, aborda-se a incorporação de ambas as noções no Brasil. Por fim, trata das implicações epistêmicas dessa nova configuração terminológica, decorrente do esforço em aproximar compreensões e debates oriundos dos campos da segurança alimentar e nutricional e da alimentação e nutrição, em sua diversidade de perspectivas sobre os problemas alimentares e nutricionais.

\section{O contexto internacional e a emergência das noções de adequado e saudável}

\section{A emergência da noção de alimentação adequada}

A alimentação foi concebida como um direito humano fundamental na década de 1940 a partir da conjuntura que envolveu as grandes guerras mundiais e as decorrentes situações de insegurança alimentar e nutricional. Assim, o Direito Humano à Alimentação foi reconhecido na Declaração Universal dos Direitos Humanos, em 1948, e consagrado no Pacto Internacional dos Direitos Econômicos, Sociais e Culturais (PIDESC), em 1966 24,25,26. Esse último é o instrumento que trata do tema de maneira mais abrangente 27 , visto que os Estados signatários assumem a responsabilidade de prover a alimentação como direito humano universal e de proteger a sua população da fome. Tal objetivo seria perseguido por meio de medidas no tocante ao provimento de alimentos, difusão de conhecimentos científicos, reforma agrária, sustentabilidade ambiental e medidas de cooperação internacional concernente à garantia da segurança alimentar mundial 28 .

Contudo, por aproximadamente cinco décadas, a segurança alimentar (e não o direito à alimentação) foi a principal pauta no discurso público. Após essa lacuna temporal, tal direito só passou a receber particular atenção quando pesquisas realizadas na década de 1980 revelaram que a insegurança alimentar dos indivíduos era principalmente um resultado da pobreza e da falta de acesso ao alimento e não da sua disponibilidade no mercado 27,29.

Foi durante a Cúpula Mundial da Alimentação realizada em 1996 que os Estados-Membros solicitaram ao Comitê de Direitos Econômicos, Sociais e Culturais, órgão fiscalizatório do PIDESC, um Comentário Geral (CG) que tivesse como objetivo identificar algumas das principais questões relacionadas ao direito à alimentação adequada. Dessa maneira, em 1999, esse Comitê explicitou o conteúdo normativo desse direito no CG no 12 dos Direitos Econômicos, Sociais e Culturais 27,29,30.

Conforme apresentado nesse CG: "O direito à alimentação adequada realiza-se quando cada homem, mulher e criança, sozinho ou em companhia de outros, tem acesso físico e econômico, ininterruptamente, à alimentação adequada ou aos meios para sua obtenção. O direito à alimentação adequada não deverá, portanto, ser interpretado em um sentido estrito ou restritivo, que o equaciona em termos de um pacote mínimo de calorias, proteínas e outros nutrientes específicos. $O$ direito à alimentação adequada terá de ser resolvido de maneira progressiva. No entanto, os estados têm a obrigação precípua de implementar as ações necessárias para mitigar e aliviar a fome, como estipulado no parágrafo 2 do artigo 11, mesmo em épocas de desastres, naturais ou não" 30 (grifos nossos).

Para o Comitê, seu conteúdo essencial consiste nos seguintes tópicos:

(1) A disponibilidade do alimento, em quantidade e qualidade suficiente para satisfazer as necessidades dietéticas das pessoas, livre de substâncias adversas e aceitável para uma dada cultura. (2) A acessibilidade ao alimento de forma sustentável e que não interfira com a fruição de outros direitos humanos 30 .

De acordo com Mechlem 27, o CG no 12 foi fortemente influenciado pelo estudo de Asbjørn Eide, relator especial para o direito à alimentação, da Subcomissão de Prevenção da Discriminação e Proteção às Minorias da Organização das Nações Unidas (ONU). Em 1987, Eide investigou de forma inovadora a natureza e o conteúdo socioeconômico desse direito e esclareceu as correspondentes obrigações nacionais e internacionais dos Estados. Portanto, assim como todos os direitos humanos, o direito à alimentação adequada implica as obrigações do Estado em respeitar, proteger e satisfazer (facilitar/prover) este direito 27.

De acordo com o documento, o "conceito de adequação" é especialmente significativo uma vez que serve para enfatizar uma série de fatores que devem ser levados em consideração para definir se alimentos ou dietas (específicas e disponíveis em uma determinada circunstância) podem ser 
considerados os mais apropriados, tendo em vista os propósitos do PIDESC. O preciso significado de adequado é em larga medida determinado por um conjunto de condições prevalecentes, dentre as quais as sociais, econômicas, culturais, climáticas e ecológicas. Vale marcar ainda que a noção de sustentabilidade está intrinsecamente ligada à noção de adequação ao incorporar a ideia de acessibilidade e disponibilidade dos alimentos a longo prazo 30.

Em suma, o CG no 12 é um documento emblemático que traz a alimentação no contexto do direito humano, adjetivada como adequada. Para tanto, o Comitê de Direitos Econômicos, Sociais e Culturais do Alto Comissariado de Direitos Humanos da ONU, para elaborar o CG no 12, reuniu um conjunto de informações sobre o direito humano à alimentação adequada, acessando relatórios dos Estados participantes datados desde 1979 30. O processo de adjetivação, portanto, vai se constituindo por meio dos diversos debates que ocorreram nas diferentes esferas institucionais e políticas, culminando posteriormente na inclusão e formalização da expressão em documentos oficiais.

\section{A emergência da noção de alimentação saudável}

Para pensar a emergência do termo alimentação saudável, é necessário resgatar a sua associação com a instituição das ciências da nutrição, a partir da qual, a centralidade do nutriente norteia as condutas e práticas alimentares. Em paralelo, as transições epidemiológica e demográfica vinculadas ao fenômeno da transição nutricional trazem ao cenário outros aspectos constitutivos da noção da alimentação saudável, tal qual é compreendida hoje.

As ciências da nutrição surgem com as descobertas e quantificação dos nutrientes dos alimentos nos laboratórios dos químicos, fisiologistas e médicos dietistas ao final do século XIX. Um dos principais objetivos era identificar os nutrientes necessários para o funcionamento normal do organismo, tendo em vista a prevenção de deficiências nutricionais. Soma-se a isso, a perspectiva ideológica de que o atendimento aos requerimentos nutricionais seria capaz de maximizar a produtividade física e intelectual 31,32,33.

Emergiu no contexto do desenvolvimento das ciências da nutrição aquilo que Gyorgy Scrinis nomeou como "reducionismo nutricional". De acordo com o autor, trata-se de um paradigma no qual a alimentação é deslocada de contextos sociais e ecológicos mais amplos, em decorrência da excessiva ênfase atribuída aos aspectos nutricionais e bioquímicos dos alimentos e do papel destes sobre a saúde. Tal concepção foi, ao longo do tempo, influenciando orientações nutricionais, regulações sobre os rótulos dos alimentos, práticas de marketing e engenharia de alimentos, além da compreensão dos indivíduos sobre a relação entre comida, saúde e corpo. Essa forma de compreender a dimensão saudável dos alimentos ganhou um estatuto de verdade e tornou-se hegemônica a despeito de outras racionalidades 33 .

Nesse cenário, ressalta-se que a transição nutricional está ligada às rápidas transformações demográficas, mudanças alimentares e alterações metabólicas observadas em nível populacional, além de um importante incremento da morbidade por doenças crônicas não transmissíveis, a exemplo do diabetes, câncer, hipertensão, cardiopatias etc, frequentemente relacionadas ao peso corporal e à alimentação ${ }^{34}$. A transição nutricional está relacionada às transformações nos padrões socioeconômicos, de agricultura e saúde, sendo a epidemia de obesidade observada no último quarto do século XX como um fenômeno global que vem ocorrendo tanto em países desenvolvidos, quanto em países em desenvolvimento 35 .

Vale marcar que, embora a dimensão do saudável no campo alimentar e nutricional já viesse sendo formada no bojo da discussão sobre a composição nutricional dos alimentos e posteriormente na busca pela superação das carências nutricionais, a expressão "alimentação saudável”, tal como conhecemos hoje, vai sendo consolidada na virada do século XXI com a emergência da epidemia de obesidade e das políticas públicas relacionadas. Assim, sua consagração aconteceu a partir da década de 1990, no período em que estudos evidenciaram o fenômeno da transição alimentar e nutricional, os quais influenciaram a proposição da Organização Mundial da Saúde (OMS) para a criação da Estratégia Global para Alimentação Saudável, Atividade Física e Saúde em 200436.

$\mathrm{Na}$ conjuntura política internacional, com as políticas neoliberais e o enfraquecimento do papel do Estado, os mercados de alimentos influenciaram fortemente a epidemia de sobrepeso/obesidade por meio da articulação "consumidor-corporação-marketing”. Tal processo ocorreu de maneiras dife- 
rentes nos variados países, mas foi ampliado especialmente após a década de 1990, quando houve o acordo global para a redução de tarifas e a abertura do mercado para as empresas, hoje reconhecidas como transnacionais 37 .

Portanto, foi com base no reconhecimento da conjuntura que favoreceu o incremento das doenças crônicas não transmissíveis que a OMS 36 propôs a estratégia global, apontando a alimentação e a atividade física como dois dos seus principais fatores de risco. Apesar da centralidade atribuída ao desequilíbrio energético na relação de causalidade das doenças crônicas não transmissíveis, a estratégia global, ao indicar o papel dos Estados na formulação de políticas e planos de ação para melhorar a alimentação, demonstrou um movimento de ampliação da visão acerca da alimentação e representou um dos primeiros documentos de âmbito internacional a consolidar a noção de "alimentação saudável".

\section{O contexto nacional e a confluência entre o saudável e o adequado no Brasil}

Revisitando os documentos oficiais mencionados, observa-se que entre as décadas de 1950 e 1970 as principais temáticas do campo da alimentação e nutrição giravam em torno de questões como desnutrição, suplementação alimentar, carências nutricionais, aleitamento materno, alimentação escolar, alimentação do trabalhador e educação nutricional. Portanto, no período histórico que vai do Governo Vargas até a Ditadura Militar ainda não apareciam as expressões "alimentação saudável" ou "alimentação adequada".

Na década de 1980 essa ausência foi mantida, tal como evidenciado no relatório da I Conferência Nacional de Alimentação e Nutrição, 8 a CNS (1986) 4 . Esse documento chamou atenção para o fato de o processo de redemocratização ter favorecido a ampliação do debate sobre as questões alimentares e nutricionais no Brasil, tais como: reforma sanitária e reforma agrária, segurança alimentar e fome, Sistema Único de Saúde (SUS) e Sistema de Vigilância Alimentar e Nutricional (SISVAN), tendo em vista tratar dos problemas alimentares e nutricionais no país.

Em documentos da década de 1990, a saber: o relatório final da I CNSA (1994), a publicação do CONSEA sobre a I CNSA (1995) 6 e a Política Nacional de Alimentação e Nutrição - PNAN (1a edição) 7, verificou-se que o adjetivo "saudável" foi evocado para qualificar noções como práticas alimentares, dieta e estilos de vida ou ainda "alimentação nutricionalmente balanceada e saudável". No entanto, a expressão "alimentação saudável", como popularmente conhecida, só passou a ser institucionalizada em 2004, aparecendo no campo da segurança alimentar e nutricional pela primeira vez no relatório da II CNSAN e no campo da saúde em 2006 na primeira versão do guia alimentar para população brasileira. Interpretamos que tal ocorrência da expressão "saudável" nos documentos da década de 1990 e documentos posteriores reflete a aproximação do Brasil com o debate internacional sobre transição alimentar e nutricional, que culminou na estratégia global coordenada pela OMS 36.

Ainda situando os documentos da primeira década do século XXI e agora iniciando a análise sobre a entrada da expressão "adequada", para posteriormente discutir a conjunção entre as expressões em questão, destaca-se que a proposição do direito humano à alimentação adequada vai aparecer fortemente associada com a noção de segurança alimentar e nutricional. O atual conceito de SAN apresentado na LOSAN (Lei no 11.346) de 2006 foi consolidado na ocasião da II CNSAN em 200426.

Sancionada pelo Presidente da República, a LOSAN representa no país um importante instrumento jurídico que marca o avanço em relação à promoção e garantia do direito humano à alimentação adequada, que se torna objetivo e meta da política de segurança alimentar e nutricional 26 . Conforme descrito no seu Art. 3o 10: "A segurança alimentar e nutricional consiste na realização do direito de todos ao acesso regular e permanente a alimentos de qualidade, em quantidade suficiente, sem comprometer o acesso a outras necessidades essenciais, tendo como base práticas alimentares promotoras de saúde que respeitem a diversidade cultural e que sejam ambiental, cultural, econômica e socialmente sustentáveis".

A LOSAN criou o Sistema Nacional de Segurança Alimentar e Nutricional (SISAN) tendo em vista assegurar o direito humano à alimentação adequada. Para o CONSEA, a sua criação "representa a sintese de um esforço coletivo e suprapartidário da luta nacional contra a fome, a pobreza e em defesa do DHAA" 38 (p. 30). No Art. 2o da LOSAN, a alimentação adequada é definida como: "... direito fundamental do ser humano, inerente à dignidade da pessoa humana e indispensável à realização dos direitos consagrados na Constituição Federal, devendo o poder público adotar as políticas e ações que façam necessárias para promover e garantir a segurança alimentar e nutricional da população" 10. 
Portanto, compreende-se que incorporar a noção de direito humano à alimentação adequada nas estratégias de segurança alimentar e nutricional e de desenvolvimento social é um caminho para reverter situações de fome, um pré-requisito para a realização dos demais direitos 26 . Como complementam Vasconcelos \& Moura 39 (p. 2), para alcançar a segurança alimentar e nutricional é necessário: “... a convergência de políticas e programas de vários setores com capacidades para promover, na dimensão individual e coletiva, o acesso à alimentação adequada, requerendo um amplo processo de descentralização, territorialização e gestão social".

Soma-se a isso a necessidade de medidas intersetoriais que devem ser implementadas por meio da responsabilização social e política das distintas esferas de governo, tendo em vista atuar sobre "os determinantes da insegurança alimentar moderada e grave, com foco nos grupos mais vulneráveis à violação do direito à alimentação adequada" 40 (p. 13).

Retomando a argumentação, até este momento observa-se a conformação da noção de alimentação saudável em paralelo à noção de alimentação adequada. No entanto, em contraposição, a expressão "alimentação adequada e saudável” aparecerá no ano seguinte, junto ao relatório da III CNSAN, em 200712 .

De acordo com o CONSEA 41, a entrada do termo "adequada" junto à noção de alimentação saudável, que já estava em circulação e legitimada no âmbito das discussões do campo da saúde, decorreu de um movimento voltado à problematização do reducionismo nutricional e à ampliação da compreensão sobre a alimentação humana para além de uma visão focada predominantemente na funcionalidade do componente nutricional dos alimentos.

O grupo de trabalho (GT) alimentação adequada e saudável do CONSEA debateu essa inclusão tomando como base o CG no 12 dos Direitos Econômicos, Sociais e Culturais (1999) 41. Conforme sinalizado, o documento esclarece que o direito humano à alimentação adequada não deve "ser interpretado em um sentido estrito ou restritivo, que o equaciona em termos de um pacote mínimo de calorias, proteinas e outros nutrientes específicos" e explicita que a aceitabilidade cultural, um de seus conteúdos essenciais, reitera os valores atribuídos aos alimentos, para além daqueles relacionados à valorização do seu conteúdo nutricional 30.

Vale destacar que no contexto brasileiro o CONSEA desempenha um significativo papel na articulação entre governo e sociedade civil, tendo o propósito de fomentar o direito humano à alimentação adequada no país. O Conselho tem a responsabilidade de assessorar a Presidência da República na formulação de políticas e na definição de orientações, tendo caráter consultivo. Criado em 1993 no contexto da campanha nacional Ação da Cidadania Contra a Fome, a Miséria e Pela Vida, o Conselho foi extinto em 1995 e recriado em 2003 no âmbito do Projeto e da Estratégia Fome Zero 42,43. No entanto, em janeiro de 2019, o Conselho foi novamente extinto pela Medida Provisória no 87044.

Assim, no âmbito do CONSEA, a discussão sobre a entrada do termo "adequada” ganhou inspiração na fundamentação própria do campo dos direitos humanos. Para apoiar essa ampliação conceitual os membros do GT alimentação adequada e saudável debateram sobre a dimensão biológica do "saudável”, dentro do qual o componente nutricional dos alimentos é fortemente valorizado, indicando que a inclusão do termo "adequada" seria relevante uma vez que abrangeria outras dimensões como cultura, prazer, hábitos, comensalidade, regionalidade, etnia, gênero, além do acesso, da sustentabilidade e da biodiversidade. Concluíram, portanto, que os termos embora considerados distintos são complementares 41 .

Ainda com base nesse relatório, destaca-se que o termo "adequada" deve abranger os aspectos do desenvolvimento econômico e social baseando-se em um modelo de produção de alimentos que promova a soberania alimentar. A alimentação e a comida devem ser entendidas em um sentido mais amplo e devem refletir a disponibilidade e a sustentabilidade (social, econômica e ambiental), considerando a diversidade cultural brasileira. Para tanto, no âmbito das discussões, a agroecologia foi designada "a referência de construção do modelo de produção de alimentos" voltada para a promoção da alimentação adequada e saudável 41.

Portanto, foi a partir da Plenária ocorrida em novembro de 2006 que o GT do CONSEA elaborou o conceito de alimentação adequada e saudável, o qual foi incorporado ao documento-base da III CNSAN realizada em 2007. Foram ainda delimitados o que denominaram como seus cinco princípios: (1) direito humano à alimentação adequada, (2) soberania alimentar e segurança alinmentar e nutricional, (3) intersetorialidade, (4) produção de alimentos com base agroecológica de forma 
sustentável e (5) cultura alimentar agregadora das dimensões biológica, simbólica, socioeconômica e religiosa ${ }^{41}$.

Nesse sentido, pode-se afirmar que a noção de alimentação adequada e saudável emergiu no contexto da redemocratização, em particular entre os anos de 2006 e 2007, por meio da articulação entre a sociedade civil e o governo. Para tanto, ressalta-se que essa noção não foi originada isoladamente no escopo da formulação das políticas.

Assim, foi a partir da III CNSAN 12 que os documentos relacionados ao campo da segurança alimentar e nutricional passaram a incorporar a expressão "alimentação adequada e saudável", com destaque para a sequência de documentos, a saber: a PNSAN (2010) 14, o Relatório final da IV CNSAN (2011) 17, o Relatório final da V CNSAN (2015) 21, o I PLANSAN (2012-2015) 22 e o II PLANSAN (2016-2019) 23.

Contudo, em paralelo às discussões do campo da segurança aliemntar e nutricional, o campo da alimentação e nutrição fez a transição da expressão "alimentação saudável" para "alimentação adequada e saudável", posteriormente. O termo "alimentação saudável" foi mantido em documentos como o Glossário Temático Alimentação e Nutrição 13 e a 3a edição da Política Nacional de Promoção da Saúde 15, e foi somente em 2012 18, com a segunda versão da PNAN, que se observou a entrada da expressão AAS. Portanto, há uma distância temporal de cinco anos entre a entrada da expressão nos documentos institucionais do campo da segurança alimentar e nutricional quando comparado ao campo da saúde.

No âmbito da PNAN 18, a alimentação adequada e saudável vem sendo definida como: "... a prática alimentar apropriada aos aspectos biológicos e socioculturais dos indivíduos, bem como ao uso sustentável do meio ambiente. Ou seja, deve estar em acordo com as necessidades de cada fase do curso da vida e com as necessidades alimentares especiais; referenciada pela cultura alimentar e pelas dimensões de gênero, raça e etnia; acessivel do ponto de vista físico e financeiro; harmônica em quantidade e qualidade; baseada em práticas produtivas adequadas e sustentáveis com quantidades mínimas de contaminantes físicos, quimicos e biológicos" 18 (p. 67).

Contudo, ressalta-se que a expressão alimentação adequada e saudável ainda não foi incorporada à segunda edição do Glossário Temático: Alimentação e Nutrição publicada em 2013 13, mas já aparece na segunda versão do Guia Alimentar para a População Brasileira de 2014 20, que busca incluir os debates do campo da segurança alimentar e nutricional na sua argumentação, sendo indutor de políticas na área, como será discutido.

De acordo com o glossário temático do Ministério da Saúde 19 (p. 15): "Alimentação saudável, fem. sin. Alimentação equilibrada. Padrão alimentar adequado às necessidades biológicas e sociais dos indivíduos e de acordo com as fases do curso da vida. Notas: i) Deve ser acessivel (física e financeiramente), saborosa, variada, colorida, harmônica e segura quanto aos aspectos sanitários. ii) Esse conceito considera as práticas alimentares culturalmente referenciadas e valoriza o consumo de alimentos saudáveis regionais (como legumes, verduras e frutas), sempre levando em consideração os aspectos comportamentais e afetivos relacionados às práticas alimentares. Em inglês: healthy eating".

Como pode ser observado, o conceito de alimentação saudável proposto pelo Ministério da Saúde não se restringe ao domínio biológico e inclui as questões socioculturais do comer, embora não inclua de forma ampliada as questões ambientais da produção de alimentos, como observado em comparação à definição da segunda versão da PNAN. Cabe lembrar que o termo saudável dialoga com a área de saúde de forma intensa e extrapola o campo da alimentação e nutrição.

Retomando a consulta documental, segundo o Guia Alimentar para a População Brasileira 20, a alimentação adequada e saudável deriva de um sistema alimentar socialmente e ambientalmente sustentável e deve estar baseada em práticas de produção adequadas e sustentáveis, trazendo uma noção ampliada sobre alimentação muito próxima ao conceituado na segunda PNAN.

A alimentação adequada e saudável é um direito humano básico que envolve a garantia ao acesso permanente e regular, de forma socialmente justa, a uma prática alimentar adequada aos aspectos biológicos e sociais do indivíduo e que deve estar em acordo com as necessidades alimentares especiais; ser referenciada pela cultura alimentar e pelas dimensões de gênero, raça e etnia; acessível do ponto de vista físico e financeiro; harmônica em quantidade e qualidade, atendendo aos princípios da variedade, equilíbrio, moderação e prazer; e baseada em práticas produtivas adequadas e sustentáveis 20 .

A versão mais atual do guia alimentar faz críticas ao reducionismo nutricional e defende a comensalidade, buscando fortalecer as dimensões sociocultural e ambiental do comer 45 , bem como a dimensão de um direito humano fundamental, o que nos remete à noção de adequação no campo 
da alimentação. Assim, o lançamento desse guia parece "consolidar" a nova terminologia, sendo uma das implicações mais expressivas da instituição histórica entre os termos, uma vez que alcança destaque internacional.

Partindo do atual guia alimentar, analisando o texto da citada publicação, pode-se afirmar que a atual proposição sobre alimentação adequada e saudável repousa na regulação do consumo dos alimentos atualmente intitulados de ultraprocessados e no estímulo ao consumo dos alimentos in natura, o que leva a uma inevitável discussão sobre a sua produção. Assim, a contemporânea proposição é influenciada pelos debates acadêmicos e institucionais sobre as dimensões socioculturais da alimentação e sobre os modernos processos de transformação no campo agroalimentar e seus impactos à saúde humana e ao meio ambiente.

Vale ressaltar que o debate em torno da categoria alimentação adequada e saudável, seja do ponto de vista conceitual, seja no sentido de sua adoção nas políticas no campo da nutrição e alimentação, é marcado por visões por vezes conflitantes e disputas de interesses que permeiam. É nesse sentido que Burlandy et al. 46, ao refletirem sobre as principais concepções, arranjos institucionais e políticas públicas relacionadas à área da alimentação e nutrição nos diversos contextos geopolíticos, trataram, de forma oportuna, que existe uma multiplicidade de termos no que se refere a esta área. Segundo os autores, a diversidade em torno das temáticas alimentares e nutricionais "reflete diferenças nas visões de mundo e nos modos de conceber os problemas e ações políticas desejáveis”.

Para os autores, o esforço voltado para análise de política deve ser compreendido como "modos de disputa” pertencentes ao campo político. Assim, eles criticam a visão de um trabalho acadêmico que seja considerado externo ao processo político e travestido de neutralidade. Tal disputa é concebida entre entendimentos, visões de mundo, projetos políticos e até mesmo distintas racionalidades dos sujeitos em questão, sejam daqueles que atuam na academia, sejam daqueles interessados no objeto da política 46 .

Sendo assim, os autores recomendam um olhar criterioso para as "descontinuidades e rupturas entre os diversos modos de pensar as questões sobre as quais se pretende intervir" 46 (p. 11). Defendem ainda que a análise de políticas precisa considerar as características dos Estados, seus modos de construção, relações e o conjunto de problemas considerados dignos de intervenção ou regulação. No caso específico deste estudo, sinalizam também a importância de analisar os seus aparatos institucionais, que de alguma maneira atuam na organização da ação estatal e marcam o processo político.

Ao discutir sobre o caráter polissêmico da noção de alimentação saudável, vale aqui trazer Azevedo 47 , pesquisadora que destaca os aspectos que contribuíram para sua construção social. Conforme destaca, compreender a proposta da alimentação na contemporaneidade, com base em uma perspectiva polissêmica, é um desafio. Segundo explica, os cursos que formam os profissionais de saúde ainda adotam o conceito reducionista, pautado nas abordagens dietoterápicas, restritivas e quantitativas. Portanto, considera que no campo da nutrição é preciso levar em consideração as premissas da sustentabilidade, possibilitando que os profissionais da área atuem de forma coerente e sintonizada com as diretrizes das políticas públicas de alimentação e nutrição 47 .

A autora destaca que as discussões sociais sobre o alimento na atualidade vão além das questões do acesso e das situações de fome e partem para preocupações sobre sua qualidade, seus componentes culturais e os aspectos socioambientais da sua produção. Assim, as discussões sobre os riscos ambientais e as repercussões do moderno sistema agroalimentar passaram a ser incluídas, e o conceito de alimento saudável e adequado passou a assumir "componentes socioambientais e culturais favorecendo a agricultura familiar e orgânica, a Agroecologia, a soberania alimentar e o local como espaço privilegiado de produção alimentar" 47 (p. 100). Salienta ainda a importância dos aspectos da transição epidemiológica e nutricional e referencia a entrada da mulher no mercado de trabalho, o aumento da longevidade, o desenvolvimento tecnológico e a crescente urbanização como componentes que também tiveram influência na evolução deste conceito. 


\section{Considerações finais}

A conjunção entre as expressões que conformaram a contemporânea noção de "alimentação adequada e saudável" no Brasil ocorre no contexto da redemocratização, sendo influenciada especialmente pelos debates desenvolvidos no âmbito do CONSEA. A terminologia genuinamente brasileira trata da confluência entre duas expressões historicamente situadas, até então tratadas por grupos particulares nas distintas esferas governamentais. Isso é, a "alimentação adequada" vinha sendo enfatizada pelo extinto Ministério do Desenvolvimento Social e Combate à Fome, e a "alimentação saudável” vinha sendo reforçada pelo Ministério da Saúde. Partindo desta análise, argumenta-se que a combinação entre as mesmas é representativa do esforço em aproximar as compreensões e os debates dos campos da segurança alimentar e nutricional e da alimentação e nutrição no que se refere à polissêmica noção de alimentação. Argumenta-se ainda que ela é representativa da forma como o país vem lidando com a dupla carga fome-sobrepeso/obesidade.

Foram apresentados os elementos motivadores da adoção da terminologia nas políticas públicas brasileiras. Atualmente, tratamos de uma compreensão ampliada sobre a noção de alimentação, que com base no debate sobre a superação do foco no componente nutricional dos alimentos inclui a compreensão sobre um direito humano fundamental, bem como a compreensão sobre as questões socioculturais e afetivas do comer e sobre as questões da sustentabilidade ambiental da produção de alimentos baseando-se em modelos produtivos pautados em princípios agroecológicos e da soberania alimentar.

Em específico, marca-se que a "alimentação saudável" como uma noção está disseminada, sendo comumente referida por profissionais de saúde e pela população. No entanto, o mesmo não é observado com a expressão "alimentação adequada", que espera-se que esteja em vias de ser popularizada na sociedade brasileira, reafirmando a importância do direito humano à alimentação. Marca-se ainda que recentes debates de cunho político com a participação de organizações internacionais discutiram a ampliação da noção de direito humano à alimentação adequada, propondo a inclusão da noção de "nutrição adequada", o que configuraria a expressão "direito humano à alimentação e à nutrição adequadas”, desencadeando repercussões normativas, políticas e práticas.

Em síntese, a expressão resultante da confluência dos termos alimentação adequada e alimentação saudável tem implicações para a luta pelos direitos humanos junto a sociedade brasileira, assim como tem impactos na formação e prática dos sujeitos envolvidos. Vale lembrar que a alimentação adequada e saudável é um tema de particular importância para nutricionistas e outros profissionais, não apenas da saúde, mas também dos campos das ciências sociais, agrárias, econômicas, da administração e do direito. Concluímos, assim, serem necessárias futuras investigações e discussões sobre as implicações da confluência entre os termos para o campo da alimentação e nutrição no Brasil, compreendendo que tal confluência é a um só tempo provocadora e provocada por tais implicações do campo. 


\section{Colaboradores}

J. B. Paiva, L. M. Magalhães, S. M. C. Santos, L. A. S. Santos e L. A. B. Trad participaram da concepção, elaboração e revisão do manuscrito.

\section{Informações adicionais}

ORCID: Janaína Braga de Paiva (0000-0001-84669075); Lílian Miranda Magalhães (0000-00034964-052X); Sandra Maria Chaves dos Santos (0000-0002-4706-0284); Lígia Amparo da Silva Santos (0000-0002-6925-6421); Leny Alves Bomfim Trad (0000-0002-8762-4320).

\section{Referências}

1. Ministério da Educação e Saúde. A conjuntura alimentar e o problema da nutrição no Brasil: Plano Geral de Trabalho 1953/54. Brasília: Ministério da Educação e Saúde; 1953.

2. Brasil. Decreto no 72.034 , de 30 de março de 1973. Institui o Programa Nacional de Alimentação e Nutrição (PRONAN). Aprova o I PRONAN e da outras providências. Diário Oficial da União 1973; 3 abr.

3. Brasil. Decreto no 77.116, de 6 de fevereiro de 1976. Estabelece diretrizes para a ação do Governo na área de Alimentação e Nutrição, aprova o Programa Nacional de Alimentação e Nutrição - PRONAN e dá outras providências. Diário Oficial da União 1976; 6 fev.

4. Ministério da Saúde. Relatório final da I Conferência Nacional de Alimentação e Nutrição - 8a Conferência Nacional de Saúde - CNS. Brasília: Ministério da Saúde; 1986.

5. Ministério da Saúde. Relatório final da I Conferência Nacional de Segurança Alimentar CNSA. Brasília: Ministério da Saúde; 1994.

6. Conselho Nacional de Segurança Alimentar e Nutricional. Publicação do Conselho Nacional de Segurança Alimentar e Nutricional sobre a I Conferência Nacional de Segurança Alimentar. Brasília: Conselho Nacional de Segurança Alimentar e Nutricional; 1995.

7. Ministério da Saúde. Política Nacional de Alimentação e Nutrição. Brasília: Ministério da Saúde; 1999.

8. Departamento de Atenção Básica, Secretaria de Atenção à Saúde, Ministério da Saúde. Política Nacional de Alimentação e Nutrição. 2a Ed. Brasília: Ministério da Saúde; 2003.

9. Ministério da Saúde. Relatório final da II Conferência Nacional de Segurança Alimentar e Nutricional - CNSAN. Brasília: Ministério da Saúde; 2004.

10. Brasil. Lei no 11.346 , de 15 de setembro de 2006. Cria o Sistema Nacional de Segurança Alimentar e Nutricional - SISAN com vistas em assegurar o direito humano à alimentação e dá outras providências. Diário Oficial da União 2006; 18 set.

\section{Agradecimentos}

Agradecemos à Fundação de Amparo à Pesquisa do Estado da Bahia (FAPESB) e à Coordenação de Aperfeiçoamento de Pessoal de Nível Superior (Capes) pelas bolsas de doutorado e sanduíche concedidas. Agradecemos também às equipes do Programa Integrado de Pesquisa e Cooperação Técnica Comunidade, Família e Saúde: Contextos, Trajetórias e Políticas Públicas, Instituto de Saúde Coletiva, Universidade Federal da Bahia (FASA/ISC-UFBA), do Núcleo de Estudos e Pesquisas em Alimentação e Cultura, Universidade Federal da Bahia (NEPACUFBA) e do SOAS Food Studies Centre, Universidade de Londres.

11. Coordenação-Geral da Política de Alimentação e Nutrição, Departamento de Atenção Básica, Secretaria de Atenção à Saúde, Ministério da Saúde. Guia alimentar para a população brasileira: promovendo a alimentação saudável. Brasília: Ministério da Saúde; 2006. (Série A. Normas e Manuais Técnicos).

12. Ministério da Saúde. Relatório final da III Conferência Nacional de Segurança Alimentar e Nutricional - CNSAN. Brasília: Ministério da Saúde; 2007.

13. Secretaria de Atenção à Saúde, Ministério da Saúde. Glossário temático: alimentação e nutrição. Brasília: Ministério da Saúde; 2008. (Série A. Normas e Manuais Técnicos).

14. Brasil. Decreto no 7.272 , de 25 de agosto de 2010. Regulamenta a Lei no 11.346 , de 15 de setembro de 2006, que cria o Sistema Nacional de Segurança Alimentar e Nutricional - SISAN com vistas a assegurar o Direito Humano à Alimentação Adequada, institui a Política Nacional de Segurança Alimentar e Nutricional - PNSAN, estabelece os parâmetros para a elaboração do Plano Nacional de Segurança Alimentar e Nutricional, e dá outras providências. Diário Oficial da União 2010; 26 ago.

15. Secretaria de Atenção à Saúde, Secretaria de Vigilância em Saúde, Ministério da Saúde. Política Nacional de Promoção da Saúde. Brasília: Ministério da Saúde; 2010.

16. Brasil. Emenda Constitucional no 64, de 4 de fevereiro de 2010. Altera o art. 6o da Constituição Federal, para introduzir a alimentação como direito social. Diário Oficial da União 2010; 4 fev.

17. Ministério da Saúde. Relatório final da IV Conferência Nacional de Segurança Alimentar e Nutricional - CNSAN. Brasília: Ministério da Saúde; 2011.

18. Departamento de Atenção Básica, Secretaria de Atenção à Saúde, Ministério da Saúde. 2 a Política Nacional de Alimentação e Nutrição. Brasília: Ministério da Saúde; 2012. (Série B. Textos Básicos de Saúde). 
19. Secretaria de Atenção à Saúde, Ministério da Saúde. Glossário temático: alimentação e nutrição. 2a Ed. Brasília: Ministério da Saúde; 2013.

20. Departamento de Atenção Básica, Secretaria de Atenção à Saúde, Ministério da Saúde. Guia alimentar para a população brasileira. 2a Ed. Brasília: Ministério da Saúde; 2014.

21. Ministério da Saúde. Relatório final da V Conferência Nacional de Segurança Alimentar e Nutricional - CNSAN. Brasília: Ministério da Saúde; 2015.

22. Câmara Interministerial de Segurança Alimentar e Nutricional. Plano Nacional de Segurança Alimentar e Nutricional (PLANSAN 2012-2015). Brasília: Câmara Interministerial de Segurança Alimentar e Nutricional; 2011.

23. Câmara Interministerial de Segurança Alimentar e Nutricional. Plano Nacional de Segurança Alimentar e Nutricional (PLANSAN 2016-2019). Brasília: Câmara Interministerial de Segurança Alimentar e Nutricional; 2017.

24. United Nations (UN). Universal Declaration of Human Rights. http://www.un.org/en/univer sal-declaration-human-rights/ (acessado em 01/Jun/2018).

25. Organização das Nações Unidas para a Educação, a Ciência e a Cultura. Declaração Universal dos Direitos Humanos. Adotada e proclamada pela resolução 217 A (III) da Assembléia Geral das Nações Unidas em 10 de dezembro de 1948. Brasília: Organização das Nações Unidas para a Educação, a Ciência e a Cultura; 1998.

26. Ação Brasileira pela Nutrição e Direitos Humanos. Direito humano à alimentação adequada no contexto da segurança alimentar e nutricional. Brasília: Ação Brasileira pela Nutrição e Direitos Humanos; 2010.

27. Mechlem K. Food security and the right to food in the discourse of the United Nations. European Law Journal 2004; 10:631-48.

28. Pacto Internacional sobre os Direitos Econômicos, Sociais e Culturais - PIDESC, 1966. http://www.unfpa.org.br/Arquivos/pacto_in ternacional.pdf (acessado em 27/Dez/2018).

29. Anthes C, De Shcutter O. The FAO as a human rights organization: advancing the right to food to promote public health. Brussels: Interdisciplinary Research Cell in Human Rights: 2017. (CRIDHO Working Paper).

30. United Nations. CESCR General Comment $\mathrm{n}$. 12: the right to adequate food (Art. 11). Adopted at the Twentieth Session of the Committee on Economic, Social and Cultural Rights, on 12 May, 1999. New York: United Nations; 1999. (Contained in Document E/C.12/1999/5).

31. Carpenter KJ. A short history of nutritional science: part 2 (1885-1912). J Nutr 2003; 133:975-84

32. Vernon J. The ethics of hunger and the assembly of society: the techno-politics of the school meal in modern Britain. Am Hist Rev 2005; 110:693-725.
33. Scrinis G. Nutritionism: the science and politics of dietary advice. New York: Columbia University Press; 2013.

34. Yates-Doerr E. Disease and modernities. In: Yates-Doerr E, editor. The weight of obesity:hunger and global health in postwar Guatemala. Berkeley: University of California Press; 2015. p. 27-54.

35. Popkin BM. The nutrition transition and its health implications in lower-income countries. Public Health Nutr 1998; 1:5-21.

36. World Health Organization. Global Strategy on diet, physical activity and health. Geneva: World Health Organization; 2004.

37. Lang T. Reshaping the food system for ecological public health. J Hunger Environ Nutr 2009; 4:315-55.

38. Conselho Nacional de Segurança Alimentar e Nutricional. A segurança alimentar e nutricional e o direito humano à alimentação adequada no Brasil: indicadores e monitoramento da Constituição de 1988 aos dias atuais. Brasília: Conselho Nacional de Segurança Alimentar e Nutricional; 2010.

39. Vasconcelos ABPA, Moura LBA. Segurança alimentar e nutricional: uma análise da situação da descentralização de sua política pública nacional. Cad Saúde Pública 2018; 34:e00206816.

40. Santos TG, Silveira JAC, Longo-Silva G, Ramires EKNM, Menezes RCE. Tendência e fatores associados à insegurança alimentar no Brasil: Pesquisa Nacional por Amostra de Domicílios 2004, 2009 e 2013. Cad Saúde Pública 2018; 34:e00066917.

41. Conselho Nacional de Segurança Alimentar e Nutricional. Grupo de Trabalho Alimentação Adequada e Saudável: relatório final. Brasília: Conselho Nacional de Segurança Alimentar e Nutricional; 2007.

42. Conselho Nacional de Segurança Alimentar e Nutricional. CONSEA. http://www4.planalto. gov.br/consea/consea-2 (acessado em 20/Ago/ 2012).

43. Ação Brasileira pela Nutrição e Direitos Humanos. Módulo 2: os direitos e as obrigações do DHAA. Brasília: Ação Brasileira pela Nutrição e Direitos Humanos; 2012.

44. Brasil. Medida Provisória no 870, de 1 de janeiro de 2019. Estabelece a organização básica dos órgãos da Presidência da República e dos Ministérios. Diário Oficial da União 2019; 1 jan.

45. Oliveira MSS, Amparo-Santos L. Food-based dietary guidelines: a comparative analysis between the Dietary Guidelines for the Brazilian Population 2006 and 2014. Public Health Nutr 2018; 21:210-7.

46. Burlandy L, Bocca C, Mattos RA. Mediações entre conceitos, conhecimento e políticas de alimentação, nutrição e segurança alimentar e nutricional. Rev Nutr 2012; 25:9-20.

47. Azevedo E. Alimentação saudável: uma construção histórica. Simbiótica: Razão e Sensibilidade 2014; (7):83-111. 


\section{Abstract}

Since the turn of this century, quality of diet is no longer described only as "healthy", but also as "adequate", in the scope of Brazilian public policies for food and nutrition and for food and nutrition security. These notions have been developed and defended socially and historically and have undergone expansions and redefinitions over time. The study proposed to analyze how the expression "adequate and healthy diet" was established in Brazil. We performed a document analysis, aimed at elucidating the conceptual development of the two terms in the Brazilian context. Institutional documents were analyzed, such as original texts from the fields of food and nutrition security and food and nutrition. A transition was observed in the way of conceiving healthy eating in the field of food and nutrition, previously focused on nutrient composition. We are currently experiencing an expanded understanding of the notion of diet, based on the debate to overcome the focus on foods' nutritional composition and including an understanding of a fundamental human right, as well as the understanding of sociocultural and affective issues in eating and issues of environmental sustainability in food production based on food production models oriented by agroecological and food sovereignty principles. The article argues that the combination of the two expressions reflects the effort to incorporate the understandings and debates in the fields of food and nutrition security and food and nutrition as regards the polysemous notion of eating.

Healthy Diet; Food and Nutrition Security; Human Rights

\section{Resumen}

En el ámbito de las politicas públicas brasileñas respecto a alimentación y nutrición, así como seguridad alimentaria y nutricional, tras la entrada en el siglo XXI, se observa que la alimentación dejó de ser denominada solamente como "saludable", $y$ se pasó también a referirse a ella como "adecuada". Estas nociones han sido construidas y defendidas socio-históricamente y han pasado por ampliaciones conceptuales y resignificaciones a lo largo del tiempo. En este estudio se propuso analizar cómo se instituyó la expresión "alimentación adecuada y saludable” en Brasil. Se realizó un análisis documental, teniendo como meta elucidar el desarrollo conceptual de los términos en cuestión dentro del contexto brasileño. Se analizaron documentos institucionales, como por ejemplo, trabajos originales en los campos de la seguridad alimentaria y nutricional y de la alimentación y nutrición. Se observa una transición en el modo de concebir la alimentación saludable en el campo de la alimentación y nutrición, antes centrada en la composición de los nutrientes. Actualmente, existe una comprensión más amplia sobre la noción de alimentación, a partir del debate sobre su superación cuando se centraba en el componente nutricional de los alimentos, que incluye la inclusión de un derecho humano fundamental, así como de cuestiones socioculturales y afectivas que conlleva comer, además de razones de sostenibilidad ambiental en la producción de alimentos, conforme a modelos productivos orientados sobre principios agroecológicos $y$ de soberanía alimentaria. Se argumenta que la combinación entre las expresiones es representativa del esfuerzo para aproximar las interpretaciones y los debates de los campos de la seguridad alimentaria y nutricional y de la alimentación y nutrición en lo que se refiere a la noción polisémica de alimentación.

Dieta Saludable; Seguridad Alimentaria e Nutricional; Derechos Humanos
Recebido em 27/Dez/2018

Versão final reapresentada em 15/Mai/2019 Aprovado em 08/Jul/2019 